\title{
On the Paper Chromatography of the Leaf Pigments I*
}

\author{
By Masukichirou AsAm1**
}

\author{
淺見盆吉郎： 綠葉色素のペーパークロマトグラフィーI
}

Since the adsorptional separation of the leaf pigments was first published by Tswett(1) in 1936, a number of successive investigation ${ }^{(2)}$ have been reported. In all of these, however, solely the so-called "column chromatographic" method was employed, and yet we have never been acquainted with any investigations based on the "paper chromatographic" method. Hence the author tried to establish a certain procedure on this subject.

\section{Experimental}

1) Preparation of sample: About $20 \mathrm{~g}$ of fresh, or 3 to $4 \mathrm{~g}$ of dried leaves of white clover (Trifolium repens L.) was extracted with $200 \mathrm{cc}$ of methanol-acetone mixture $(3: 1)$ for $24 \mathrm{hrs}$, in the dark and cool, in order to prevent any deterioration of the leaf pigments. The filtered extract was evaporated under reduced pressure below $25^{\circ} \mathrm{C}$, and the dried residual pigment mixture was then dissolved in methanol-acetone to prepare $0.62 \%$ of the matter. ( $8 \mu 1$ of this solution contains $50 \gamma$ of the sample.)

2) Apparatus for development: The employed apparatus for ascending paper chromatography is illustrated in Fig. 1. For preparing paper strips, a sheet of filter paper (Toyo Filter Paper Co's No. 50 for paper chromatography) was cut into rectangular size $(1.2 \times 35 \mathrm{~cm})$, and the original spot was uniformly settled on each strip at the point $5 \mathrm{~cm}$ from the bottom.

\section{Results}

1) Determination of the developing agents: Various solvents were used in this trial (Table 1). It followed that the aromatic hydrocarbons and $\mathrm{CCl}_{4}$ were the most suitable for the development of leaf pigments, and they gave almost identical results either anhydrous or saturated with water. When the development was carried

* The outline of this communication was read at the Temporary Meeting of the Agricultural Chemical Society of Japan, Nov. 4th 1951, which was held at the Kyushu Univ., and the short article was published in Japanese in the Journal of the Shiga Pref. Jun. Coll., Ser. A, Vol. 1 No. 4 (1951), p. 139.

** Laboratory of Domestic Science, Junior College of Shiga Pref., Hikone City. 
out with these solvents, two green spots of chlorophyll $\mathrm{a}$ and $\mathrm{b}^{*}$, and three yellow spots of carotinoids were clearly separated in every case. The unmoved greenish brown colored spots was probably due to the secondary degeneration product of leaf pigments, because its colora. tion became deeper after decrepitude of the sample.

2) Effect of temperature: The temperature effect on the development of leaf pigments with toluene and $\mathrm{CCl}_{4}$ was shown in Fig. 2. With rise of temperature, spots of carotenoids drew nearer to each other, while that of chlorophylls suffered no significant influences. This might account for the probable denaturation of carotenoids caused by atmospheric oxidation. As a rule, constant results can not be obtainable, when the development was carried out above $30^{\circ} \mathrm{C}$.

\section{3) Correlations among quantity of} specimen, time of development and $R_{f}$ value: During the development, carotenoids behaved in somewhat different manner from chlorophylls (Fig. 3,4); the spots of carotenoids ran by almost same
Fig. 1. Apparatus for development.

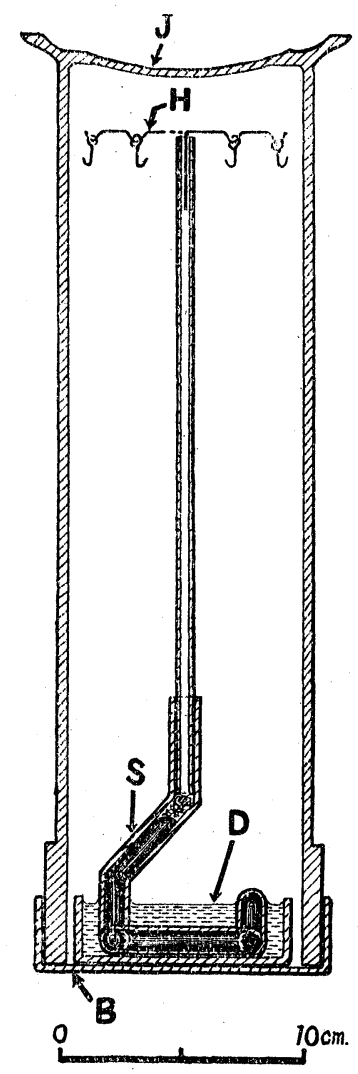

$\mathrm{J}$ : Jacket cylidder.

$\mathrm{H}$ : Strip hanger. (stainless steel)

$\mathrm{D}$ : Solvent distributor.

S : Hanger stand.

B: Base dish. ratio of the progressing solvent front, so that their $R_{f}$ values, like as the case of ordinary "partition chromatography," tended toward nearly constant after a certain time had passed and a slight influence of difference of the sample quantity was seen. In contrast, spots of chlorophylls moved rather rapidly in earlier stage of development, but through gradual diminuation, reached almost unmovable at last, irrespective of the extension of solvent front; wherefore, the change of their $R_{f}$ values showed an asymptotic degeneration with the progressive development. In addition, the distance of movement of chlorophylls became greater according to the increase

\footnotetext{
* Doubling of them were mostly seen when the development was carried out with aromatic hydrocarbons.
} 
Table 1. Results of Development by means of various Solvents.

(Quantify of Sample; $100 \gamma$, at $15-22^{\circ} \mathrm{C}$.)

\begin{tabular}{|c|c|c|c|c|c|c|c|c|c|}
\hline \multirow{2}{*}{ 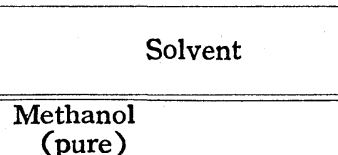 } & \multirow{2}{*}{$\begin{array}{c}\begin{array}{c}\text { Solvent } \\
\text { Front }\end{array} \\
8.4 \mathrm{~cm}\end{array}$} & \multicolumn{8}{|c|}{$\begin{array}{l}\text { Rf Values and Colors* } \\
\text { (Mean of each three Tests) }\end{array}$} \\
\hline & & - & - & - & $\begin{array}{l}0.40 \\
(\mathrm{OY})\end{array}$ & $\begin{array}{l}0.61 \\
(G)\end{array}$ & $\begin{array}{l}0.73 \\
(Y)\end{array}$ & $\begin{array}{c}0.81 \\
(Y G)\end{array}$ & - \\
\hline $\begin{array}{c}\text { Methanol } \\
(80 \%)\end{array}$ & 8.6 & $\begin{array}{l}0.00 \\
(Y)\end{array}$ & $\begin{array}{l}0.11 \\
(\mathrm{G})\end{array}$ & - & $\begin{array}{c}0.28 \\
(\mathrm{OY})\end{array}$ & - & $\begin{array}{l}0.52 \\
(Y)\end{array}$ & $\begin{array}{l}0.79 \\
\text { (OY) }\end{array}$ & - \\
\hline $\begin{array}{c}\text { Ethanol } \\
\text { (pure) }\end{array}$ & 9.7 & - & - & - & - & - & $\begin{array}{l}0.63 \\
(\mathrm{OY})\end{array}$ & $\begin{array}{l}0.88 \\
(\mathrm{G})\end{array}$ & $\begin{array}{c}0.98 \\
(\mathrm{GY})\end{array}$ \\
\hline $\begin{array}{l}\text { Ethanol } \\
(80 \%)\end{array}$ & 10.5 & - & - & - & - & - & - & $\begin{array}{l}0.86 \\
(G)\end{array}$ & $\begin{array}{c}0.98 \\
(\mathrm{Y})\end{array}$ \\
\hline $\begin{array}{l}\text { Methanol: } \\
\text { Ethanol }(1: 1)\end{array}$ & 9.4 & - & 一 & - & - & $\begin{array}{c}0.61 \\
(\mathrm{OY})\end{array}$ & $\begin{array}{l}0.78 \\
(\mathrm{G})\end{array}$ & $\begin{array}{l}0.89 \\
(\mathrm{Y})\end{array}$ & $\begin{array}{c}0.99 \\
(\mathrm{GY})\end{array}$ \\
\hline $\begin{array}{l}\text { n-Butanol } \\
\text { (anhydrous) }\end{array}$ & 9.3 & $=$ & - & - & - & - & - & - & $\begin{array}{l}0.98 \\
(\mathrm{G})\end{array}$ \\
\hline $\begin{array}{l}\text { n-Butanol } \\
\text { (satrd. with water) }\end{array}$ & 9.4 & - & - & - & - & - & - & - & $\stackrel{0.97}{(G)}$ \\
\hline $\begin{array}{l}\text { i-Butanol } \\
\text { (anhydrous) }\end{array}$ & 9.6 & - & - & - & - & - & - & - & $\begin{array}{l}0.96 \\
(\mathrm{G})\end{array}$ \\
\hline $\begin{array}{l}\text { i-Butanol } \\
\text { (satrd. with water) }\end{array}$ & 9.4 & - & - & - & - & - & - & $\begin{array}{l}0.88 \\
(G)\end{array}$ & $\begin{array}{l}0.98 \\
(Y)\end{array}$ \\
\hline $\begin{array}{l}\text { Ethanol: } \\
\quad \text { n-Butanol }(1: 1)\end{array}$ & 9.8 & - & - & - & - & - & - & - & $\begin{array}{l}0.95 \\
(\mathrm{G})\end{array}$ \\
\hline Cyclohexanol & 5.9 & - & - & - & - & - & - & - & $\begin{array}{l}0.95 \\
(\mathrm{G})\end{array}$ \\
\hline Acetone & 8.3 & - & - & - & - & - & - & $\begin{array}{l}0.79 \\
(\mathrm{Y})\end{array}$ & $\begin{array}{l}0.98 \\
(G)\end{array}$ \\
\hline $\begin{array}{l}\text { Benzene } \\
\text { (anhydrous) }\end{array}$ & 9.8 & $\begin{array}{l}0.00 \\
(\mathrm{GBr})\end{array}$ & - & - & $\begin{array}{l}0.22 \\
(\mathrm{YG})\end{array}$ & $\begin{array}{l}0.34 \\
(\mathrm{BG}) \\
\end{array}$ & $\begin{array}{l}0.75 \\
(\mathrm{Y})\end{array}$ & $\begin{array}{l}0.91 \\
(\mathrm{Y})\end{array}$ & $\begin{array}{l}0.99 \\
(\mathrm{OY})\end{array}$ \\
\hline $\begin{array}{l}\text { Benzene } \\
\text { (satrd. with water) }\end{array}$ & 10.3 & $\begin{array}{l}0.00 \\
(\mathrm{GBr})\end{array}$ & - & - & $\begin{array}{l}0.22 \\
(\mathrm{YG})\end{array}$ & $\begin{array}{l}0.34 \\
(\mathrm{BG})\end{array}$ & $\begin{array}{l}0.78 \\
(\mathrm{Y})\end{array}$ & $\begin{array}{l}0.89 \\
(\mathrm{Y})\end{array}$ & $\begin{array}{l}0.99 \\
(\mathrm{OY})\end{array}$ \\
\hline $\begin{array}{l}\text { Toluene } \\
\text { (anhydrous) }\end{array}$ & 10.4 & $\begin{array}{l}0.00 \\
(\mathrm{GBr})\end{array}$ & $\begin{array}{l}0.12 \\
(\mathrm{YG})\end{array}$ & $\begin{array}{l}0.16 \\
(\mathrm{BG})\end{array}$ & $\begin{array}{c}0.22 \\
(\mathrm{YG})\end{array}$ & $\begin{array}{l}0.39 \\
(\mathrm{BG})\end{array}$ & $\begin{array}{l}0.69 \\
(Y)\end{array}$ & $\begin{array}{l}0.85 \\
(Y)\end{array}$ & (OY) \\
\hline $\begin{array}{l}\text { Toluene } \\
\text { (satrd. with water) }\end{array}$ & 10.4 & $\begin{array}{c}0.00 \\
(\mathrm{GBr})\end{array}$ & $\begin{array}{l}0.14 \\
(\mathrm{YG})\end{array}$ & $\begin{array}{l}0.19 \\
(\mathrm{BG})\end{array}$ & $\begin{array}{l}0.23 \\
(\mathrm{YG})\end{array}$ & $\begin{array}{l}0.36 \\
(\mathrm{BG})\end{array}$ & 0.72 & (Y) & (OY) \\
\hline $\begin{array}{l}\text { Xylene } \\
\text { (anhydrous) }\end{array}$ & 10.2 & $\begin{array}{l}0.00 \\
(\mathrm{GBr})\end{array}$ & $\begin{array}{l}0.11 \\
(\mathrm{YG})\end{array}$ & $\begin{array}{l}0.18 \\
\text { (BG) }\end{array}$ & $\begin{array}{l}0.23 \\
(\mathrm{YG})\end{array}$ & $\begin{array}{l}0.38 \\
(\mathrm{BG})\end{array}$ & $\begin{array}{l}0.70 \\
(Y)\end{array}$ & 0.84 & (OY) \\
\hline $\begin{array}{l}\text { Xylene } \\
\text { (satrd. with water) }\end{array}$ & 9.8 & $\begin{array}{l}0.00 \\
(\mathrm{GBr})\end{array}$ & - & - & $\begin{array}{l}0.23 \\
(\mathrm{YG})\end{array}$ & $\begin{array}{l}0.35 \\
(\mathrm{BG})\end{array}$ & $\begin{array}{l}0.35 \\
(\mathrm{Y})\end{array}$ & $\begin{array}{l}0.87 \\
(\mathrm{Y})\end{array}$ & $\begin{array}{l}0.99 \\
(\mathrm{OY})\end{array}$ \\
\hline $\begin{array}{l}\mathrm{Cl}_{4} \mathrm{Cl}_{4} \\
\text { (anhydrous) }\end{array}$ & 7.7 & $\begin{array}{c}0.00 \\
(\mathrm{GBr})\end{array}$ & - & - & $\begin{array}{l}0.15 \\
(\mathrm{YG})\end{array}$ & $\begin{array}{l}0.20 \\
(\mathrm{BG})\end{array}$ & $\begin{array}{l}0.40 \\
(\mathrm{Y})\end{array}$ & $\begin{array}{l}0.76 \\
(\mathrm{Y})\end{array}$ & $\begin{array}{l}0.98 \\
(\mathrm{OY})\end{array}$ \\
\hline $\begin{array}{l}\mathrm{C} \mathrm{Cl}_{4} \\
\text { (satrad. with water) }\end{array}$ & 7.3 & $\begin{array}{l}0.00 \\
(\mathrm{GBr})\end{array}$ & - & - & $\begin{array}{l}0.14 \\
(\mathrm{YG})\end{array}$ & $\begin{array}{l}0.17 \\
(\mathrm{BG})\end{array}$ & $\begin{array}{l}0.35 \\
(\mathrm{Y})\end{array}$ & $\begin{array}{l}0.72 \\
(\mathrm{Y})\end{array}$ & (OY) \\
\hline $\begin{array}{l}\mathrm{CHCl}_{3} \\
\text { (anhydrous) }\end{array}$ & 9.0 & - & - & - & - & - & - & $\begin{array}{l}0.95 \\
(G)\end{array}$ & $\begin{array}{l}0.99 \\
(Y)\end{array}$ \\
\hline $\begin{array}{l}\mathrm{CHCl}_{3} \\
\text { (satrd. with water) }\end{array}$ & 8.3 & - & - & - & - & - & - & $\begin{array}{l}0.96 \\
(\mathrm{G})\end{array}$ & $\begin{array}{l}0.99 \\
(\mathrm{Y})\end{array}$ \\
\hline $\begin{array}{l}\text { Phenol** } \\
(80 \%)\end{array}$ & 6.6 & - & - & - & - & - & - & - & $\begin{array}{c}0.99 \\
(\mathrm{DG})\end{array}$ \\
\hline Lutidine & 8.3 & - & - & - & - & - & $\begin{array}{l}0.68 \\
(\mathrm{Y})\end{array}$ & - & 0.98 \\
\hline Collidine & 7.4 & - & - & - & - & - & 一 & - & $\begin{array}{l}0.98 \\
\text { (G) }\end{array}$ \\
\hline $\begin{array}{l}\text { Benzine } \\
\left.\text { (bp. } 45-60^{\circ} \mathrm{C}\right)\end{array}$ & 10.4 & $\begin{array}{l}0.00 \\
(\mathrm{G})\end{array}$ & $\begin{array}{l}0.05 \\
(\mathrm{Y})\end{array}$ & - & - & - & - & - & - \\
\hline $\begin{array}{l}\text { Benzene: } \\
\text { Benzine }(1: 1)\end{array}$ & 9.6 & - & $\begin{array}{l}0.03 \\
(\mathrm{YG})\end{array}$ & $\begin{array}{r}0.20 \\
(\mathrm{BG})\end{array}$ & - & - & $\begin{array}{l}0.24 \\
(\mathrm{Y})\end{array}$ & $\begin{array}{l}0.30 \\
(\mathrm{Y})\end{array}$ & $\begin{array}{c}0.99 \\
(\mathrm{OY})\end{array}$ \\
\hline
\end{tabular}

* Y : yellow, OY : orange-yellow, GY : greenish yellow, YG : yellowish green, BG : blueish green, DG : dark green, GBr: greenish brown.

** Operated in coal-gas stream. 
Fig. 2. Effect of temperature.

Quantity of sample: 100. $\gamma$.

Time for development: ca. 20 minutes.

a) Developed with toluene

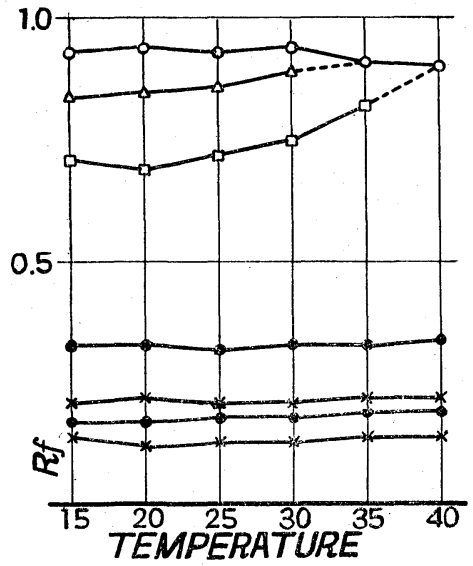

Signature of the spots

$$
\left\{\begin{array}{l}
O \text { carotenoid (orange yellow) } \\
\triangle \text { carotenoid (yellow) }
\end{array}\right.
$$

Fig. 3. Progressive change of the spots in the course of develpoment.

a) Developed with toluene, Quantity of sample: $100 \gamma$.

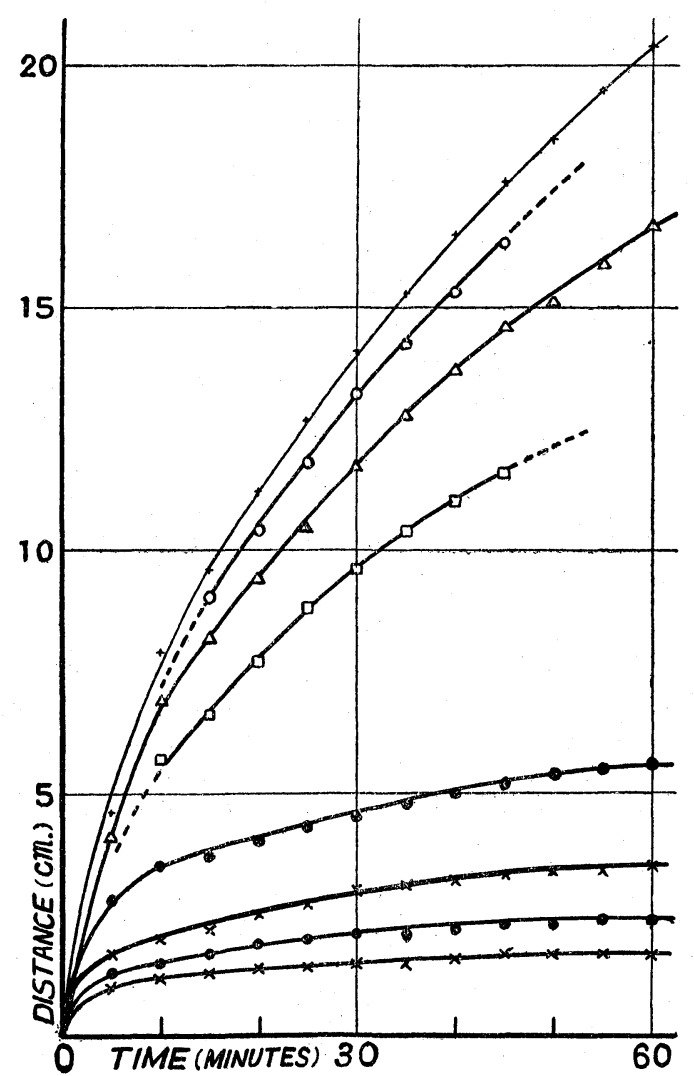

b) Developed with $\mathrm{CCl}_{4}$

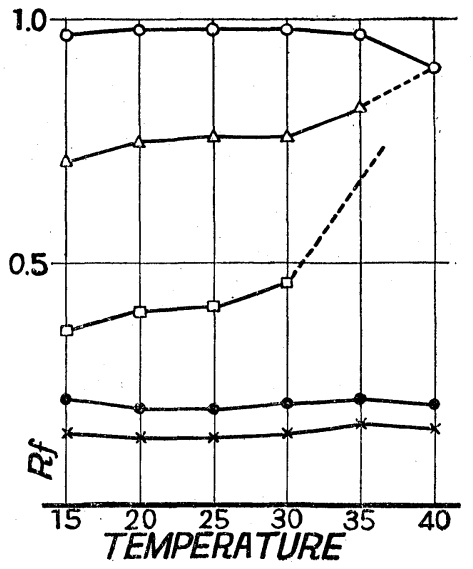

Chlorophyll a × Chlorophyll b

b) Developed with toluene ; quantity of sample: $300 \gamma$.

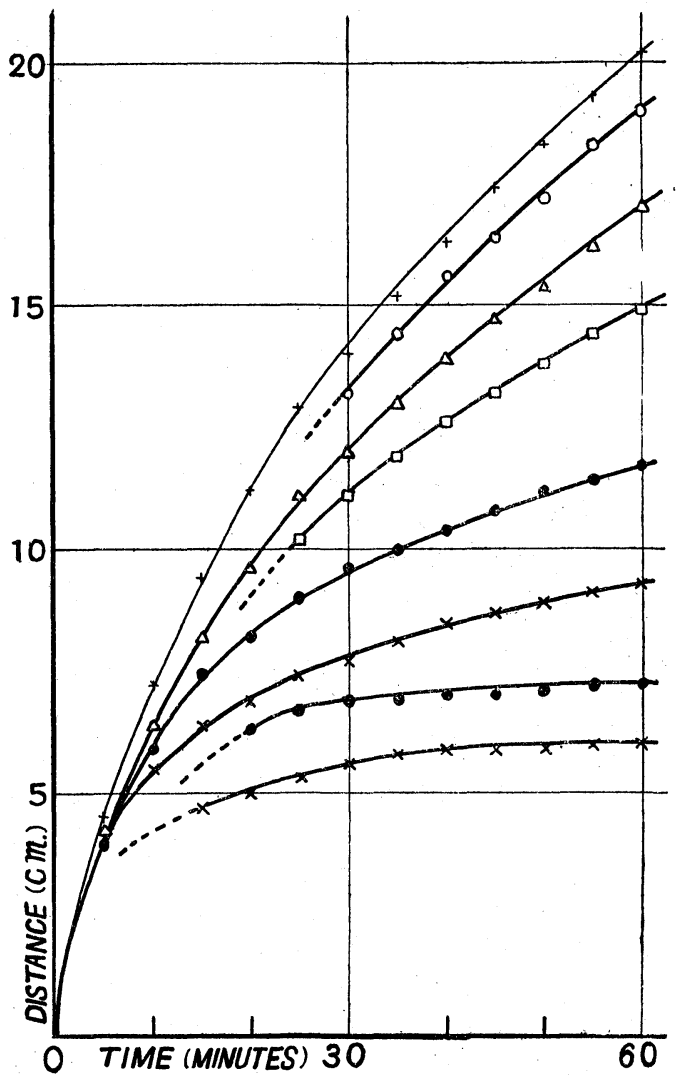

+ : Solvent front. 
c) Developed with $\mathrm{CCl}_{4}$, Quantity of sample : $100 \gamma$.

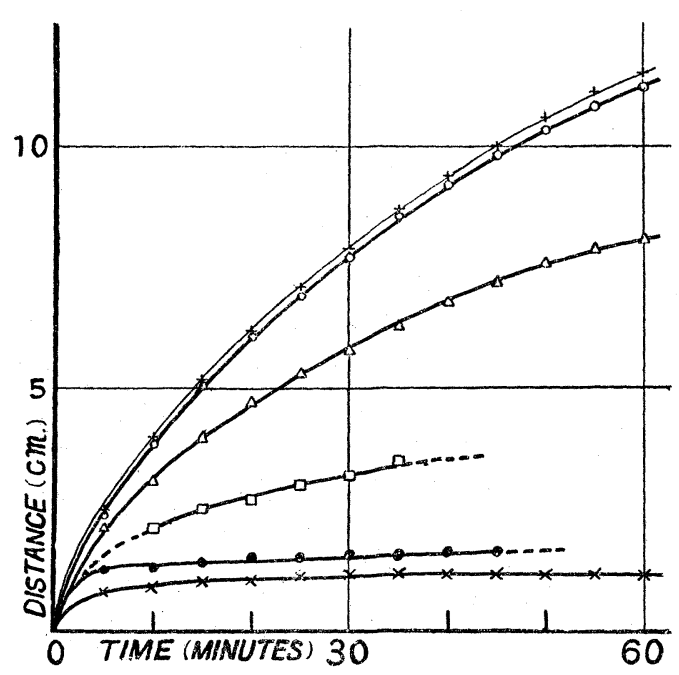

Fig. 4. Change of the $R_{f}$ values.

a) Developed with toluene, Quantity of sample: $100 \gamma$

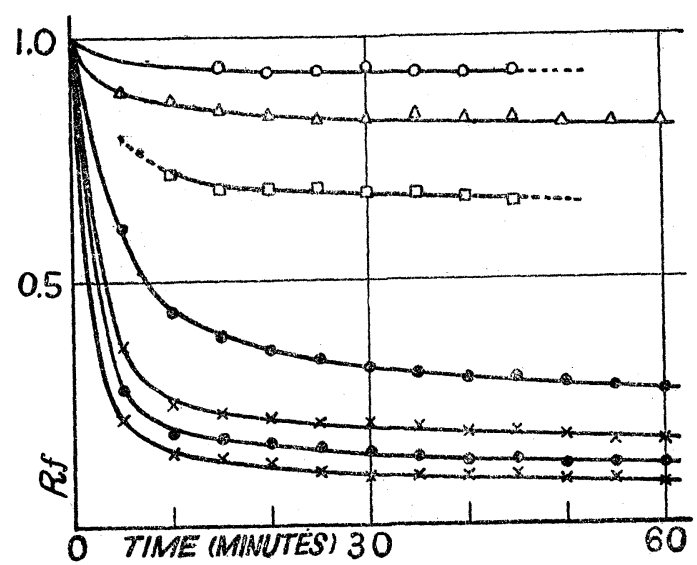

c) Developed with $\mathrm{CCl}_{4}$,

Quantity of sample: $100 \gamma$.

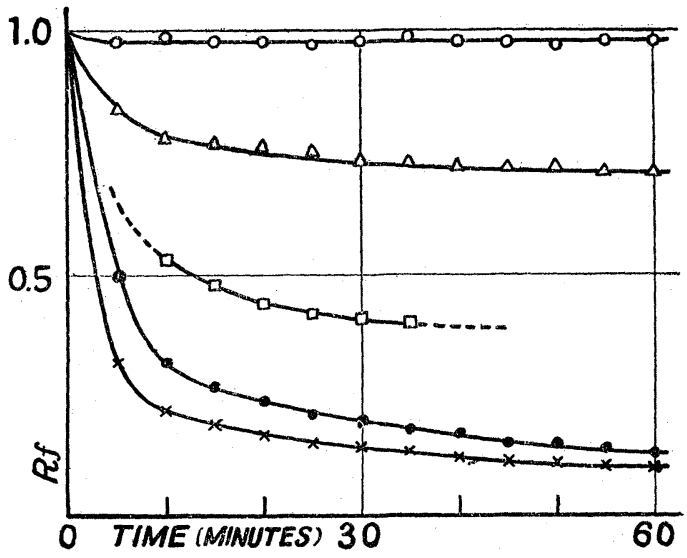

d) Developed with $\mathrm{CCl}_{4}$, Quantity of sample: $300 \gamma$.

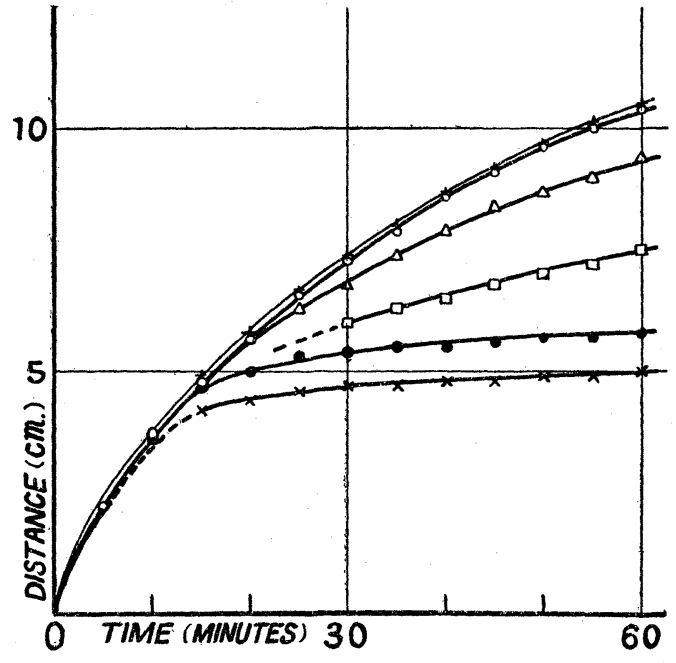

b) Developed withtoluene, Quantity of sample : $300 \gamma$.

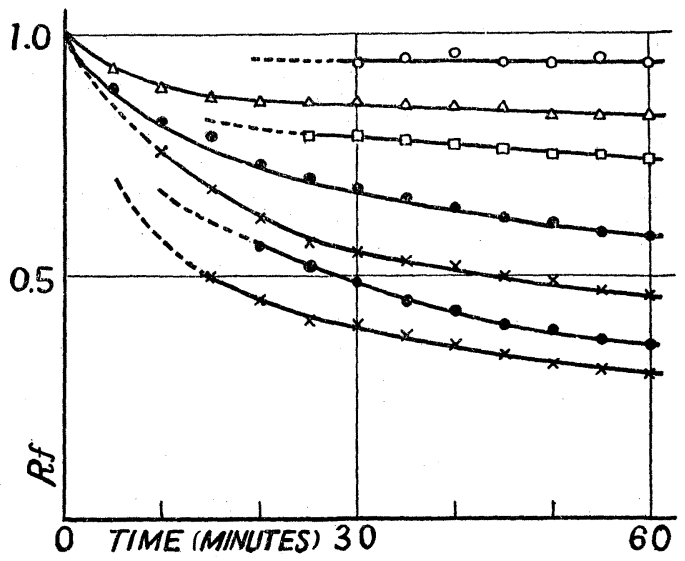

d) Developed with $\mathrm{CCl}_{4}$, Quantity of sample: $300 \gamma$.

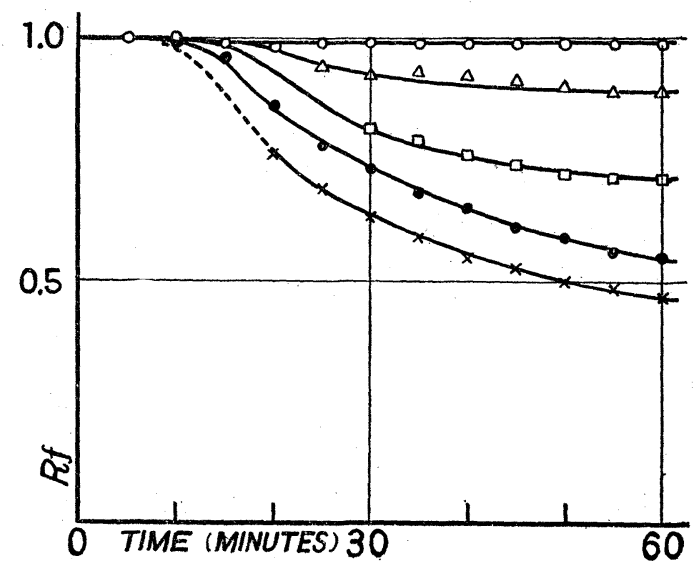


Fig. 5. Correlations between $R_{f}$ values of cnlorophyils and the quantity of sample. $\left(20^{\circ} \mathrm{C}\right.$.)

a) Developed with toluene.

b) Developed with $\mathrm{CCl}_{4}$.
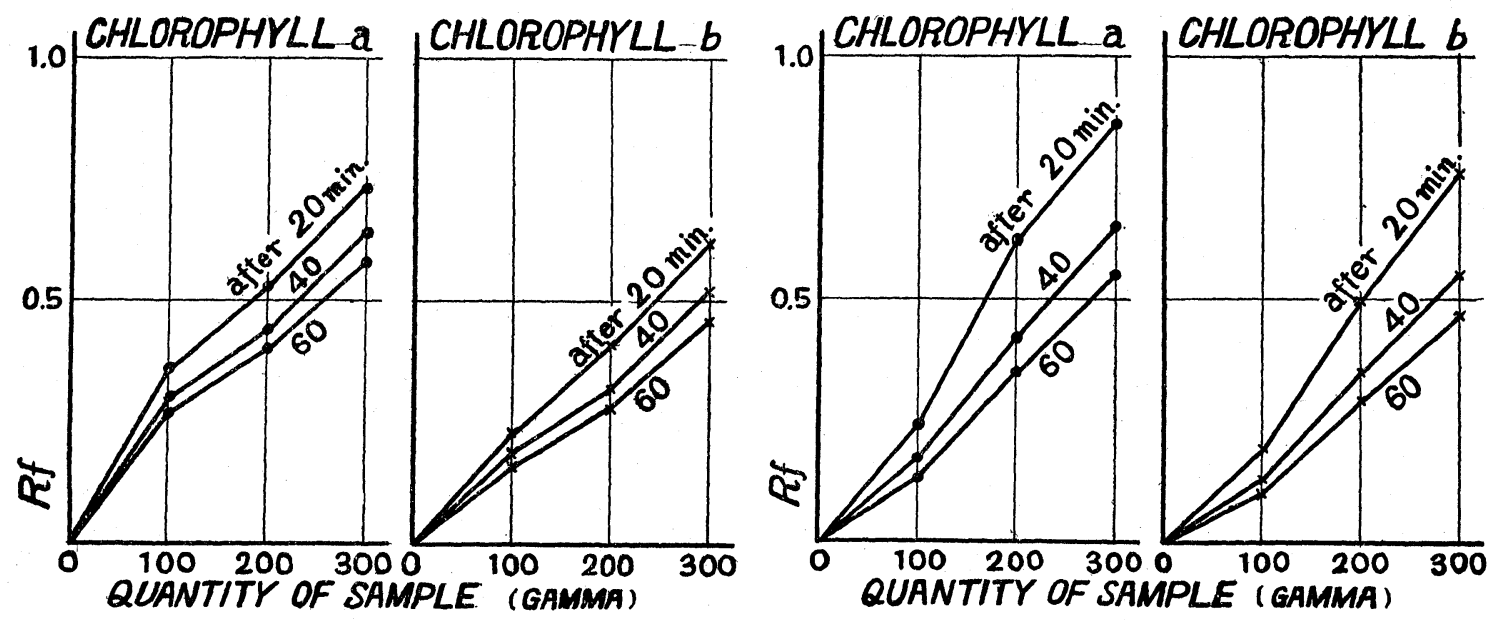

of sample quantity. These correlations are shown in Fig. 5, which were drawn up as follows; based on the results of Fig. 4, the corresponding $R_{f}$ values of colorophylls were plotted at every same moment inthe course of development, when the sample quantity was 100,200 and $300 \gamma$ respectively. Relying upon these figures, the relative change of $R_{f}$ values of Chlorophylls corresponding to the sample quantities was approximately rectilinear, in every case.

\section{Considerations}

Strain et al.(3) have already discussed in detail on the behaviors of the leaf pigments during the development by means of column chromatographic method. According to their investigations, the developed status of these pigments were remarkably influenced by many factors, especially on contamination of some colorless impurities (alcohols, amides, fatty acids etc.) in the chromatographic system. The sample which was employed at present investigation, was indeed very crude. It might contain much of the impurities effective on chromatographic results, so that the data revealed on this report are no more valuable but some special examples under some complicated conditions. Datailed investigations are therefore, in progress on the behaviors of purified pigments, and also on the influences of several conditioning factors. At present, it may be that these preliminary informations will be of some value for a convenient separation of the leaf pigments, and further, for establishment of a quantitative estimation procedure of chlorophylls, from the results, which are given in Fig. 5. 
Cordial gratitude is expressed here, for Mr. T. Chiba, Biological Institute of Kyūshū Univ., of his encouragement and helpful suggestions, and also for Miss $M$. Saito, of her kind assistance on this investigation.

\section{Literatures cited}

1) Tswett, M., Ber. d. Deut. botan. Ges., 24, 384 (1906).

2) Strain, H. H., "Chromatographic adsorption analysis." Interscience Publishers, Inc., New York, N.Y. (1942).

3) Strain, H. H., Manning, W. M., and Hardin, G., Biol. Bull., 86, 169 (1944).

Strain, H. H., Ind. Eng. Chem., Anal. Ed. 18, 605 (1946); ibid., 42, 1307 (1950).

Strain, H. H., Anal. Chem., 21, 75 (1949): ibid., 22, 41 (1950).

\section{総括}

1） ベンゼン系炭化水素並びに四塩化炭素は 綠葉色素のペーパー・クロマトグラフーに最 も適した展開凨であり,シロッメクサ葉よりメタノール・アセトン混液を以て抽出した粗色素 存試料とし,クロロフィル $\mathrm{a}, \mathrm{b}$ 及び 3 種のカロチノィドを明膫に分離し得る。

2）この方法による展開は $30^{\circ} \mathrm{C}$ 以下で実施しなければカロチノィドの班点に相当の攪乱 を受ける。

3）クロロフィルの展開曲線はカロチノィドのそれと性格を異にし，展開時間の経過に件 つて漸次勾配の減衰が著しくなる。

4）クロロフィルの Rf 值は原点の試料量の增加に件つて直線的に錬大する。 